\title{
Análise de eventos hidrológicos extremos, usando-se a distribuição GEV e momentos LH
}

\author{
Manoel M. F. de Queiroz ${ }^{1}$ \& Fazal H. Chaudhry ${ }^{2}$
}

\begin{abstract}
RESUMO
A distribuição de probabilidade generalizada de valores extremos (GEV), tem facilitado muitas aplicações em hidrologia, utilizada na modelação de eventos extremos naturais. Estudos sobre o assunto mostram que estimadores de máxima verossimilhança dos parâmetros da GEV são instáveis em pequenas amostras, podendo fornecer valores absurdos do parâmetro de forma, quando então são recomendados estimadores de momentos $\mathrm{LH}$, baseados na combinação linear de estatísticas de altas ordens, introduzidas para caracterizar a parte mais alta da distribuição e os valores extremos dos dados; contudo, não se dispõe de programas computacionais para PC, que modelem eventos extremos via momentos LH. Objetivou-se, com este trabalho, apresentar a modelação de eventos hidrológicos extremos através da distribuição GEV, utilizando-se momentos LH para estimar seus parâmetros e o teste estatístico proposto por Wang (1998) para verificação da qualidade dos ajustes desenvolvidos no ambiente Matlab. Como resultados, são apresentados as estimativas dos parâmetros da GEV, os valores das taxas de momentos LH: coeficientes de variação, assimetria e curtose, e os valores do teste de qualidade de ajuste, em aplicações com dados de vazão de rios do Paraná.
\end{abstract}

Palavras-chave: análise de freqüência, estimação, teste de hipótese, eventos extremos

\section{Analysis of extreme hydrological events using GEV distribution and LH moments}

\begin{abstract}
The generalized extreme-value (GEV) distribution has facilitated many applications in hydrology, used to model a wide variety of natural extreme events. Previous studies show that small-sample maximum-likelihood estimators parameters are unstable and demonstrates that absurd values of the GEV shape parameter can be generated. It is recommended that LH moments estimators, based on linear combinations of higher-order statistics, should be introduced for characterizing the upper part of distributions and larger events in data. However, there have been no computer packages for PC that model extreme events by LH moments. The objective of this paper was to present the modeling of hydrological extreme events by GEV distribution, using LH moments to estimate its parameters and the goodness-of-fit test proposed by Wang (1998) to evaluate the goodness-of-fit, both developed in Matlab. The results are presented for the estimation of the parameters of the GEV, the values coefficient of variation, skewness, kurtosis and goodness-of-fit test values, and fitting extreme flow observed in Paraná Rivers by GEV distribution.
\end{abstract}

Key words: frequency analysis, estimation, hypothesis testing, extreme events

${ }^{1}$ Centro de Ciências Exatas e Tecnológicas/UNIOESTE. Rua Universitária 2069, Jardim Universitário, CEP 85819-110, Cascavel, PR. Fone: (45)3220-3262. E-mail: mfqueiroz@unioeste.br.

${ }^{2}$ Departamento de Hidráulica e Saneamento/EESC/USP. CP 359, CEP 13565-905, São Carlos, SP. Fone (16)3274-9266, Fax (16)3274- 9212. E-mail: fazal@sc.usp.br 


\section{INTRODUÇÃO}

A distribuição de probabilidade generalizada de valores extremos (GEV), introduzida por Jenkinson (1955), que combina os três possíveis tipos de distribuição de valores extremos em uma única forma, vem sendo utilizada para representar a distribuição de valores extremos em diferentes campos, principalmente em análise de freqüência de cheias, com considerável aceitação para descrever fluxo máximo de cheias anuais. Na prática, a distribuição GEV é usada para modelar uma extensa variedade de extremos naturais, como cheias, chuvas, velocidade do vento, temperaturas e outros extremos (Martins \& Stedinger, 2000; Queiroz, 2002).

Procedimentos computacionais para estimação dos parâmetros da GEV, utilizando-se estimadores de máxima verossimilhança, foram propostos por Otten \& van Montfort (1980), Prescott \& Walden (1980) e Hosking (1985), porém esses estimadores são muito instáveis quando aplicados em pequenas amostras (Hosking et al., 1985) e podem gerar valores absurdos do parâmetro de forma (k) da GEV (Martins \& Stedinger, 2000).

Recomendam-se estimadores de momentos LH, uma generalização de momentos de combinações lineares de estatísticas de ordens - momentos L (Hosking, 1990), baseados na combinação linear de estatísticas de altas ordens, introduzidas para caracterizar, também, a parte mais alta da distribuição e os valores extremos dos dados, através das ordens LH mais altas, com vistas à estimação de eventos com grandes períodos de retorno (Wang, 1997). A ordem LH igual a zero é similar a momentos L; assim, a utilização de momentos LH possibilita o ajuste da distribuição GEV aos dados amostrais, desde a sua forma descritiva feita por momentos L até a caracterização dos valores mais altos, para uma análise preditiva. Desta forma, e se considerando a performance e versatilidade do Matlab, desenvolveu-se um algoritmo que realiza o ajuste da distribuição GEV a dados amostrais de valores extremos, através de 5 níveis de combinação lineares das estatísticas de ordens LH $(0,1,2,3$ e 4$)$ sobre os mesmos. Aplicou-se o referido procedimento para modelar séries de cheias anuais e de vazões mínimas de sete dias de 88 estações fluviométricas, localizadas em rios do Paraná.

\section{MATERIAL E MÉTODOS}

\section{Distribuição GEV}

A função de distribuição generalizada de valores extremos - GEV, que engloba as três formas assintóticas de distribuição de valores extremos conhecidas como valor extremo do tipo I (VEI), valor extremo do tipo II (VEII) e valor extremo do tipo III (VEIII) (Fisher \& Tippett, 1928; Gumbel, 1958), é definida, segundo Jenkinson (1955), como segue:

$$
F(x)=P(X \leq x)=\exp \left[-\left(1-k \frac{x-u}{\alpha}\right)^{\frac{1}{k}}\right]
$$

sendo

$-\infty<x<+\infty, \mathrm{k}=0$ - distribuição VEI (Distribuição Gumbel) $\varepsilon \leq x<+\infty, \mathrm{k}<0-$ distribuição VEII

$-\infty<x \leq+\omega, \mathrm{k}>0-$ distribuição VEIII

onde u é um parâmetro de posicionamento com $-\infty<\mathrm{u}<+\infty$, $\alpha$ é um parâmetro de escala com $0<\alpha<+\infty$ e k é um parâmetro de forma com $-\infty<\mathrm{k}<+\infty$; desta forma, quando $\mathrm{k}>$ 0 , o limite superior da distribuição assintótica VEIII se torna $\omega=\mathrm{u}+\alpha / \mathrm{k}$ e, quando $\mathrm{k}<0$, o limite inferior da distribuição assintótica VEII se torna $\varepsilon=u+\alpha / \mathrm{k}$.

O p-ésimo quantil da distribuição GEV é dado pela seguinte relação, decorrente da Eq. 1:

$$
\mathrm{x}_{\mathrm{p}}=\mathrm{u}+\frac{\alpha}{\mathrm{k}}\left[1-(-\ln (\mathrm{p}))^{\mathrm{k}}\right], 0<\mathrm{p}<1
$$

Combinando a distribuição de Gumbel com a sua variável reduzida $(\mathrm{z}): \mathrm{z}=(\mathrm{x}-\mathrm{u}) / \alpha$, obtém-se $\mathrm{F}(\mathrm{x})=\exp [-\exp (-\mathrm{z})]$ que resulta em:

$$
\mathrm{z}=\ln [-\ln (\mathrm{F}(\mathrm{x}))]
$$

A variável reduzida de Gumbel também se relaciona com o período de retorno $(\mathrm{T}), \mathrm{T}=1 / \mathrm{F}(\mathrm{x})$; $\log$, a Eq. 3 pode ser usada para definir $\mathrm{z}$ com respeito às distribuições VEI, VEII e VEIII; assim, em um gráfico x versus z, define-se o comportamento das três formas de distribuição de valores extremos, com relação à posição de plotagem de x, como mostra a Figura 1.

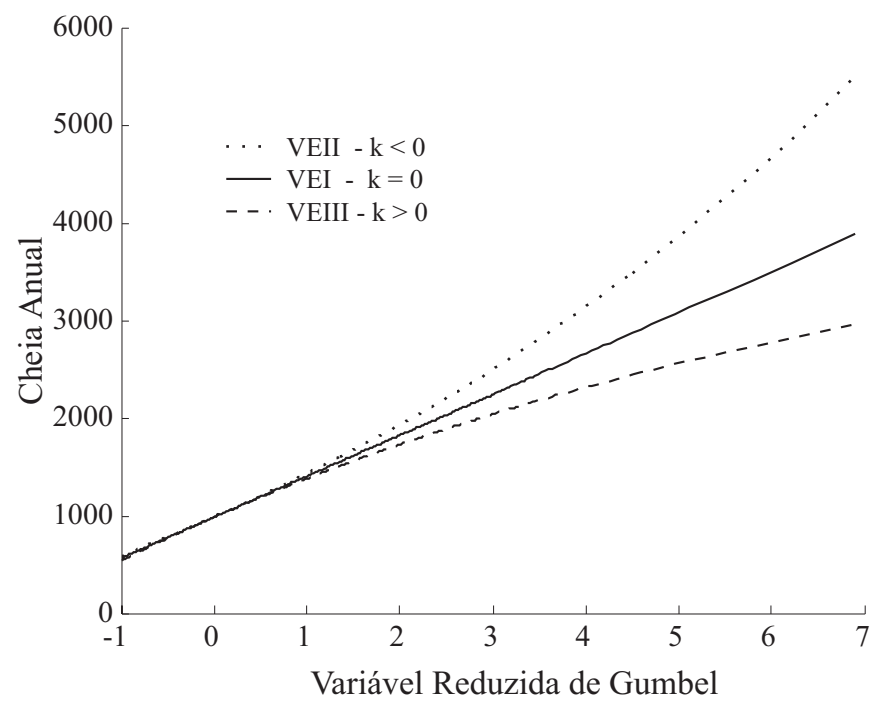

Figura 1. Distribuição das três formas de valores extremos representados pela GEV

\section{Momentos LH}

Seja $X_{1}, X_{2}, \ldots, X_{n}$ uma amostra aleatória de uma população com função densidade de probabilidade $\mathrm{f}(\mathrm{x})$ e função distribuição $\mathrm{F}(\mathrm{x})$, e $\mathrm{x}_{1: \mathrm{n}} \leq \mathrm{x}_{2: \mathrm{n}} \leq \cdots \leq \mathrm{x}_{\mathrm{n}: \mathrm{n}}$ as estatísticas de ordem obtidos da amostra acima, o valor esperado do i-ésimo menor valor da variável é dado através da seguinte expressão (Hosking, 1990). 


$$
E\left[X_{i: n}\right]=\frac{n !}{(n-i) !(i-1) !} \int_{0}^{1} x[F(x)]^{i-1}[1-F(x)]^{n-i} d F(x)
$$

Dada uma amostra de tamanho $\mathrm{n}$, retirada de uma distribuição $\mathrm{F}(\mathrm{x})=\mathrm{P}(\mathrm{X} \leq \mathrm{x})$ e com base na combinação linear das mais elevadas estatísticas de ordem e na Eq. 4, os momentos LH são definidos como:

$$
\begin{aligned}
& \lambda_{1}^{\eta}=\mathrm{E}\left[\mathrm{X}_{(\eta+1):(\eta+1)}\right] \\
& \lambda_{2}^{\eta}=\frac{1}{2} \mathrm{E}\left[\mathrm{X}_{(\eta+2):(\eta+2)}-\mathrm{X}_{(\eta+1):(\eta+2)}\right] \\
& \lambda_{3}^{\eta}=\frac{1}{3} \mathrm{E}\left[\mathrm{X}_{(\eta+3):(\eta+3)}-2 \mathrm{X}_{(\eta+2):(\eta+3)}+\mathrm{X}_{(\eta+1):(\eta+3)}\right] \\
& \lambda_{4}^{\eta}=\frac{1}{4} \mathrm{E}\left[\mathrm{X}_{(\eta+4):(\eta+4)}-3 \mathrm{X}_{(\eta+3):(\eta+4)}+3 \mathrm{X}_{(\eta+2):(\eta+4)}-\mathrm{X}_{(\eta+1):(\eta+4)}\right]
\end{aligned}
$$

em que $\lambda_{1}^{\eta}$, maior valor esperado na amostra de tamanho $\eta+1$, corresponde a uma medida de posicionamento da distribuição; $\lambda_{2}^{\eta}$, metade da diferença entre os maior e segundo maior valores esperados na amostra de tamanho $\eta+2$, caracteriza a expansão da parte superior da distribuição; $\lambda_{3}^{\eta}$, reflete como está a assimetria da parte superior da distribuição, através dos três maiores valores esperados na amostra de tamanho $\eta+3$ e $\lambda_{4}^{\eta}$ provê uma medida da pontiagudez da parte superior da distribuição, através dos quatros maiores valores esperados na amostra de tamanho $\eta+4$.

Quando $\eta=0$, momentos LH se tornam iguais aos momentos L; quando $\eta$ aumenta, os momentos LH refletem mais e mais as características da parte superior da distribuição e dos valores extremos máximos dos dados. Momentos LH são chamados momentos $\mathrm{L}_{1}$, momentos $\mathrm{L}_{2}, \ldots$ para $\eta=1,2, \ldots$ respectivamente. Normalizando os momentos LH, obtém-se o coeficiente de variação LH $\left(\tau_{2}^{\eta}\right)$, assimetria $\left(\tau_{3}^{\eta}\right)$ e curtose $\left(\tau_{4}^{\eta}\right)$, respectivamente, como:

$$
\tau_{2}^{\eta}=\frac{\lambda_{2}^{\eta}}{\lambda_{1}^{\eta}} ; \quad \tau_{3}^{\eta}=\frac{\lambda_{3}^{\eta}}{\lambda_{2}^{\eta}} ; \quad \tau_{4}^{\eta}=\frac{\lambda_{4}^{\eta}}{\lambda_{2}^{\eta}}
$$

ordenando a amostra em $\mathrm{x}_{(1)} \leq \mathrm{x}_{(2)} \leq \ldots \ldots \ldots . . \mathrm{x}_{(\mathrm{n})}$, a estimativa dos momentos LH é feita como segue:

$$
\begin{aligned}
& \hat{\lambda}_{1}^{\eta}=\frac{1}{{ }^{n} C_{\eta+1}} \sum_{i=1}^{n}{ }^{i-1} C_{\eta} x_{(i)} \\
& \hat{\lambda}_{2}^{\eta}=\frac{1}{2} \frac{1}{{ }^{n} C_{\eta+2}} \sum_{i=1}^{n}\left({ }^{i-1} C_{\eta+1}-{ }^{i-1} C_{\eta}{ }^{n-1} C_{1}\right) \cdot x_{(i)} \\
& \lambda_{3}^{\eta}=\frac{1}{3} \frac{1}{{ }^{n} C_{\eta+3}} \sum_{i=1}^{n}\left({ }^{i-1} C_{\eta+2}-2^{i-1} C_{\eta+1}{ }^{n-1} C_{1}+{ }^{i-1} C_{\eta}{ }^{n-i} C_{2}\right) \cdot x_{(i)} \\
& \hat{\lambda}_{4}^{\eta}=\frac{1}{4} \frac{1}{{ }^{n} C_{\eta+4}} \sum_{i=1}^{n}\left({ }^{i-1} C_{\eta+3}-3^{i-1} C_{\eta+2}{ }^{n-1} C_{1}+3^{i-1} C_{\eta+1}{ }^{n-i} C_{2}-{ }^{i-1} C_{\eta}{ }^{n-1} C_{3}\right) \cdot x_{(i)}
\end{aligned}
$$

em que

$$
{ }^{m} C_{j}=\left(\begin{array}{l}
m \\
j
\end{array}\right)=\frac{m !}{j !(m-j) !} \quad \text { e }{ }^{m} C_{j}=0 \text { quando } j>m
$$

\section{Estimativas dos parâmetros da distribuição GEV}

Dada uma amostra, os três parâmetros $\mathrm{k}, \alpha$ e u da distribuição GEV podem ser estimados considerando-se a estimativa dos momentos LH amostrais, através das Eqs. 10, 11, 12 e 13, para um valor selecionado de $\eta$ e $\kappa \neq 0$, como segue (Wang, 1997):

$$
\begin{aligned}
\lambda_{1}^{\eta} & =\mathrm{u}+\frac{\alpha}{\kappa}\left[1-\Gamma(1+\mathrm{k})(\eta+1)^{-k}\right] \\
\lambda_{2}^{\eta}= & \frac{(\eta+2) \alpha \Gamma(1+\kappa)}{2 ! \kappa}\left[-(\eta+2)^{-\kappa}+(\eta+1)^{-\kappa}\right] \\
\lambda_{3}^{\eta}= & \frac{(\eta+3) \alpha \Gamma(1+\kappa)}{3 ! \kappa}\left[-(\eta+4)(\eta+3)^{-\kappa}+2(\eta+3)(\eta+2)^{-\kappa}\right)\left(-(\eta+2)(\eta+1)^{-\kappa}\right] \\
\lambda_{4}^{\eta}= & \frac{(\eta+4) \alpha \Gamma(1+\kappa)}{4 ! \kappa}\left[-(\eta+6)(\eta+5)(\eta+4)^{-\kappa}+3(\eta+5)(\eta+4)(\eta+3)^{-\kappa}\right) \\
& \left(-3(\eta+4)(\eta+3)(\eta+2)^{-\kappa}+(\eta+3)(\eta+2)(\eta+1)^{-\kappa}\right]
\end{aligned}
$$

assim, os parâmetros u, $\alpha$ e k da distribuição GEV podem então ser estimados substituindo-se os três primeiros momentos LH nas Eqs.14, 15 e 16 pelos seus respectivos estimadores amostrais nas Eqs.10, 11 e 12, para cada valor de h selecionado (Wang, 1997).

Para facilitar o procedimento computacional, Wang (1997) propôs uma equação aproximada para o cálculo de $\mathrm{k}$, tomando como base as Eqs. 15 e 16 e a Eq. 9 que definem $\tau_{3}^{\eta}$, a qual corresponde a $\mathrm{k}=\alpha_{0}+\alpha_{1}\left[\tau_{3}^{\eta}\right]+\alpha_{2}\left[\tau_{3}^{\eta}\right]^{2}+\alpha_{3}\left[\tau_{3}^{\eta}\right]^{3}$, donde os coeficientes $\alpha_{0}, \alpha_{2}$ e $\alpha_{3}$ variam em função de $\eta$ (Tabela 1 ). Uma vez obtido o valor de k, as Eqs. 15 e 14 fornecem, respectivamente $\alpha$ e u.

Tabela 1. Valores dos coeficientes $\alpha_{0}, \alpha_{2}$ e $\alpha_{3}{ }^{*}$

\begin{tabular}{ccccc}
\hline$\eta$ & $\alpha_{0}$ & $\alpha_{1}$ & $\alpha_{2}$ & $\alpha_{3}$ \\
0 & 0,2849 & $-1,8213$ & 0,8140 & $-0,2835$ \\
1 & 0,4823 & $-2,1494$ & 0,7269 & $-0,2103$ \\
2 & 0,5914 & $-2,2351$ & 0,6442 & $-0,1616$ \\
3 & 0,6618 & $-2,4548$ & 0,5733 & $-0,1273$ \\
4 & 0,7113 & $-2,5383$ & 0,5142 & $-0,1027$ \\
\hline *Wang (1997) & & & &
\end{tabular}

Análises de dados observados e dados obtidos via simulação Monte Carlo, mostraram que momentos LH reduzem as influências indesejáveis que os menores eventos amostrais podem exercer na estimação de eventos com grandes períodos de retorno, comparado ao uso de momentos L (Wang, 1997; Queiroz, 2002).

\section{Razões de momentos e coeficiente de variação LH}

As razões de momentos e coeficiente de variação $\mathrm{LH}$ da distribuição GEV são calculadas combinando-se as relações em 9 com as Eqs. 14, 15, 16 e 17; já as razões de momentos LH e coeficiente de variação LH amostrais são obtidos relacionando-se os momentos LH amostrais, indicados através das Eqs. 10, 11, 12 e 13, seguindo-se as fórmulas em 9, cujos momentos LH da distribuição GEV são substituídos por seus respectivos estimadores. 
Teste de qualidade de ajuste da distribuição GEV via momentos LH

A distribuição GEV pode ser ajustada para uma série de dados, igualando-se os seus três primeiros momentos LH aos respectivos momentos LH amostrais, como já indicado (Wang, 1997). A curtose LH da população $\left(\tau_{4}^{\eta}\right)$ é uma função da assimetria LH populacional $\left(\tau_{3}^{\eta}\right)$, em que ambos dependem apenas do parâmetro de forma $\mathrm{k}$. Como o valor estimado da curtose LH amostral $\left(\hat{\tau}_{4}^{\eta}\right)$ não é usado no ajuste da distribuição GEV, Wang (1998) considerou este parâmetro para desenvolver a estatística do teste de qualidade de ajuste; deste modo e dado um particular estimador amostral $\hat{\tau}_{3}^{\eta}$, precisa-se conhecer $\mathrm{p}\left(\hat{\tau}_{4}^{\eta} \mid \hat{\tau}_{3}^{\eta}\right)$ porém não é tão simples encontrar $\mathrm{p}\left(\hat{\tau}_{4}^{\eta} \mid \hat{\tau}_{3}^{\eta}\right)$ quando a mesma depende de $\tau_{3}^{\eta}$ da população; contudo, dado $\hat{\tau}_{3}^{\eta}$, é possível inferir $\tau_{3}^{\eta}$ populacional, usando-se o teorema de Bayes, mostrado a seguir:

$$
\mathrm{p}\left(\tau_{3}^{\eta} \mid \hat{\tau}_{3}^{\eta}\right) \propto \mathrm{p}\left(\hat{\tau}_{3}^{\eta} \mid \tau_{3}^{\eta}\right) \mathrm{p}\left(\tau_{3}^{\eta}\right)
$$

donde $\mathrm{p}\left(\tau_{3}^{\eta}\right)$ é uma distribuição a priori que pode ser informativa ou não informativa. então, dado $p\left(\tau_{3}^{\eta} \mid \hat{\tau}_{3}^{\eta}\right)$ encontra-se:

$$
\mathrm{p}\left(\hat{\tau}_{4}^{\eta} \mid \hat{\tau}_{3}^{\eta}\right)=\int \mathrm{P}\left(\hat{\tau}_{4}^{\eta} \mid \tau_{3}^{\eta}, \hat{\tau}_{3}^{\eta}\right) \mathrm{P}\left(\tau_{3}^{\eta} \mid \hat{\tau}_{3}^{\eta}\right) \mathrm{d} \tau_{3}^{\eta}
$$

sendo $p\left(\hat{\tau}_{3}^{\eta} \mid \tau_{3}^{\eta}\right)$ em Eq. 18 e $p\left(\hat{\tau}_{4}^{\eta} \mid \tau_{3}^{\eta}, \hat{\tau}_{3}^{\eta}\right)$ em Eq. 19 podem ser derivadas de $p\left(\hat{\tau}_{4}^{\eta}, \hat{\tau}_{3}^{\eta} \mid \tau_{3}^{\eta}\right)$, principalmente utilizando-se simulação Monte Carlo; portanto, teoricamente é possível se comparar $\hat{\tau}_{4}^{\eta} \operatorname{com} p\left(\hat{\tau}_{4}^{\eta} \mid \hat{\tau}_{3}^{\eta}\right)$ para inferir se a distribuição subjacente é significativamente diferente da distribuição GEV; apesar disso, proceder por tal inferência requer esforço computacional que, em geral, não é prático (Wang, 1998).

Wang (1998) desenvolveu um teste de qualidade de ajuste da GEV com base em $p\left(\hat{\tau}_{4}^{\eta} \mid \tau_{3}^{\eta}=\hat{\tau}_{3}^{\eta}\right)$ como uma aproximação de $p\left(\hat{\tau}_{4}^{\eta} \mid \tau_{3}^{\eta}, \hat{\tau}_{3}^{\eta}\right)$, além de assumir que as distribuições de $\hat{\tau}_{3}^{\eta}$ e de $\hat{\tau}_{4}^{\eta}$ da GEV, seguem uma distribuição conjunta normal. Para descrição completa da distribuição conjunta normal, precisa-se conhecer a média, o desvio padrão e o coeficiente de correlação dos estimadores amostrais $\hat{\tau}_{3}^{\eta}$ e $\hat{\tau}_{4}^{\eta}$; suas médias são assumidas para representar os valores populacionais de $\tau_{3}^{\eta}$ e $\tau_{4}^{\eta}$, respectivamente, em que é negligenciado algum erro de estimação. Os desvios padrão e coeficiente de correlação, denotados como $\sigma\left(\hat{\tau}_{3}^{\eta}\right), \sigma\left(\hat{\tau}_{4}^{\eta}\right)$ e $\rho\left(\hat{\tau}_{3}^{\eta}, \hat{\tau}_{4}^{\eta}\right)$, respectivamente, são funções de $\tau_{3}^{\eta}$ e do tamanho da amostra e podem ser encontrados através de simulação Monte Carlo.

A distribuição condicional amostral de $\hat{\tau}_{4}^{\eta}$, quando $\tau_{3}^{\eta}=\hat{\tau}_{3}^{\eta}$ é normalmente distribuída com média $\tau_{4}^{\eta}$ e com desvio padrão, dado como segue (Wang, 1998):

$$
\sigma\left(\hat{\tau}_{4}^{\eta} \mid \hat{\tau}_{3}^{\eta}=\tau_{3}^{\eta}\right)=\sigma\left(\hat{\tau}_{4}^{\eta}\right)\left[1-\rho^{2}\left(\hat{\tau}_{3}^{\eta}, \hat{\tau}_{4}^{\eta}\right)\right]^{\frac{1}{2}}
$$

Um teste de hipótese de que uma série de dados vem da distribuição ajustada, pode ser conduzido na base da estimativa amostral $\hat{\tau}_{4}^{\eta}$ através da comparação da seguinte estatística (Wang, 1998):

$$
\mathrm{Z}_{\mathrm{W}}=\frac{\hat{\tau}_{4}^{\eta}-\tau_{4}^{\eta}}{\sigma\left(\hat{\tau}_{4}^{\eta} \mid \hat{\tau}_{3}^{\eta}=\tau_{3}^{\eta}\right)}
$$

com valores críticos de uma distribuição normal padrão.

O desvio padrão em Eq. 21, $\sigma\left(\hat{\tau}_{4}^{\eta} \mid \hat{\tau}_{3}^{\eta}=\tau_{3}^{\eta}\right)$, é função de $\tau_{3}^{\eta}$ e do tamanho da amostra e pode ser calculado com $\sigma\left(\hat{\tau}_{4}^{\eta}\right)$ e $\rho\left(\hat{\tau}_{3}^{\eta}, \hat{\tau}_{4}^{\eta}\right)$ através da Eq. 20, usando-se simulação Monte Carlo. Para evitar o enorme esforço computacional envolvido nas várias fases do teste, Wang (1998) propôs a seguinte aproximação:

$$
\sigma^{2}\left(\hat{\tau}_{4}^{\eta} \mid \hat{\tau}_{3}^{\eta}=\tau_{3}^{\eta}\right)=\frac{\mathrm{b}}{\mathrm{n}}+\frac{\mathrm{c}}{\mathrm{n}^{2}}
$$

donde:

$$
\mathrm{b}=\mathrm{b}_{0}+\mathrm{b}_{1}\left[\tau_{3}^{n}\right]+\mathrm{b}_{2}\left[\tau_{3}^{\eta^{n}}\right]^{2}+\mathrm{b}_{3}\left[\tau_{3}^{\eta^{n}}\right]^{3}+\mathrm{b}_{4}\left[\tau_{3}^{\eta_{3}}\right]^{4} \quad \text { e } \quad c=c_{0}+\mathrm{c}_{1}\left[\tau_{3}^{n}\right]+\mathrm{c}_{2}\left[\tau_{3}^{n}\right]^{2}+\mathrm{c}_{3}\left[\tau_{3}^{n}\right]^{3}+\mathrm{c}_{4}\left[\tau_{3}^{n}\right]^{4}
$$

os coeficientes $b_{0}, b_{1}, b_{2}, b_{3}, b_{4}, c_{1}, c_{2}, c_{3}$ e $c_{4}$ variam com os valores de $\eta$ e estão apresentados na Tabela 2 .

A Eq. 22 tem a mesma forma daquela utilizada por Chowdhury et al. (1991) para coeficiente de variação L e assimetria L e assegura que a variância é assintótica e inversamente proporcional ao tamanho da amostra, enquanto o segundo termo assegura tal efeito em amostras pequenas.

O diagrama de momentos LH também permite avaliar-se, graficamente, a qualidade dos ajustes da distribuição GEV, comparando-se os coeficientes teóricos e amostrais de assimetria e curtose. O diagrama de momentos LH tem a vantagem de possibilitar a comparação dos ajustes de diversas séries de dados, em um mesmo gráfico.

Rotina em Matlab para momentos LH e teste de Wang (1998)

Os procedimentos de ajuste da distribuição GEV através de momentos LH (Wang, 1997) e do teste de qualidade de ajuste para a mesma, proposto por Wang (1998), estão sistematizados através da seguinte rotina em Matlab, que procede ao ajuste para qualquer tamanho de amostra.

Tabela 2. Valores dos coeficientes $b_{i}$ e $c_{i}\left(i_{i=0,1,2,3 \text { e } 4}\right)$ das expressões de b e c da Eq 22*

\begin{tabular}{ccccccccccc}
\hline$\eta$ & $\mathbf{b}_{\mathbf{0}}$ & $\mathbf{b}_{1}$ & $\mathbf{b}_{2}$ & $\mathbf{b}_{3}$ & $\mathbf{b}_{4}$ & $\mathbf{c}_{\mathbf{0}}$ & $\mathbf{c}_{1}$ & $\mathbf{c}_{2}$ & $\mathbf{c}_{3}$ & $\mathbf{c}_{4}$ \\
0 & 0,0745 & 0,0555 & 0,0067 & $-0,3090$ & 0,2240 & 1,0100 & $-0,0282$ & $-2,9336$ & 4,0801 & $-1,0874$ \\
1 & 0,0579 & $-0,0328$ & 0,1524 & $-0,4102$ & 0,2672 & 1,3403 & $-0,8291$ & $-3,8777$ & 9,5371 & $-5,7866$ \\
2 & 0,0488 & $-0,0527$ & 0,1620 & $-0,3856$ & 0,2566 & 1,8800 & $-2,2233$ & $-2,5825$ & 10,4350 & $-7,3887$ \\
3 & 0,0380 & $-0,0309$ & 0,0354 & $-0,1233$ & 0,0878 & 2,6784 & $-4,8418$ & 3,5255 & 2,3736 & $-3,2076$ \\
4 & 0,0241 & 0,0024 & $-0,0813$ & 0,0733 & $-0,0210$ & 3,7793 & $-8,3485$ & 11,5170 & $-7,9095$ & 1,9459 \\
\hline${ }^{*}$ Wang (1998) & & & & & & & & & &
\end{tabular}


function $\left[\mathrm{k}, \alpha, \mathrm{u}, \mathrm{cv}, \mathrm{ca}, \mathrm{cc}, \mathrm{z}_{\mathrm{w}}\right]=\mathrm{fmomlh}(\mathrm{x})$

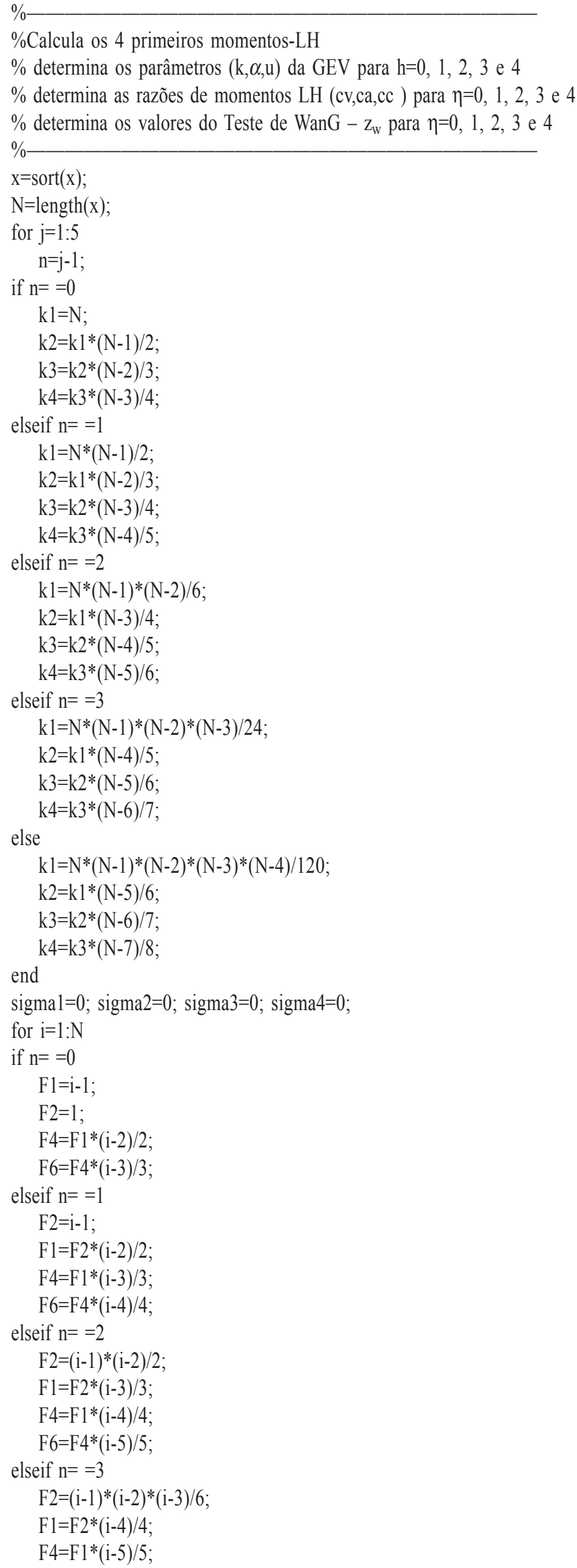

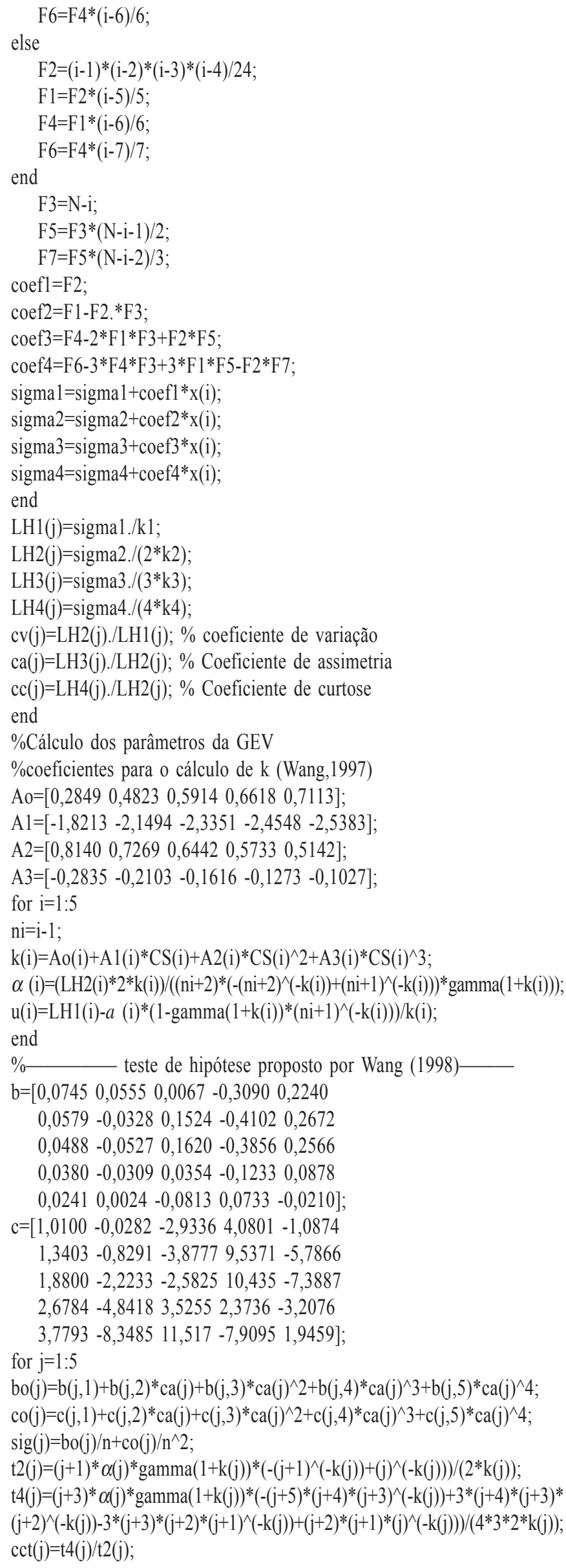

R. Bras. Eng. Agríc. Ambiental, v.10, n.2, p.381-389, 2006. 
$z(j)=a b s(\operatorname{cc}(j)-\operatorname{cct}(j)) /(\operatorname{sig}(j))^{\wedge} 0.5 ;$

end

$\%$

\section{Ajustes de cheias anual e vazões mínima de 7 dias, obser- vadas em rios do Paraná}

Séries de cheia anual e vazão mínima de 7 dias observadas em 42 estações fluviométricas instaladas em rios da subbacia 64, da Bacia hidrográfica do Paraná, estado do Paraná, foram ajustadas através da distribuição GEV e momentos LH, aplicando-se a rotina em matlab proposta. A análise de aderência foi feita utilizando-se os testes de Wang (1998) e de Kolmogorov-Smirnov, com 5\% de significância para os ajustes das séries de cheia anual e o teste de KolmogorovSmirnov, com significância de 5\%, para os ajustes de vazão mínima de 7 dias. A qualidade dos ajustes foi também avaliada através do diagrama de momentos LH, para os dois casos de séries de dados observados.

\section{RESULTADOS E DISCUSSÃO}

O algoritmo proposto realiza a estimação dos três parâmetros da distribuição GEV através de momentos LH, calcula as taxas de momentos LH e coeficiente de variação e os valores do teste de qualidade de ajuste, segundo Wang (1998). Os resultados do processo de ajuste da GEV a uma série de dados de cheias anuais ou de vazões mínimas, podem ser apresentados para os diferentes valores de $\mathrm{h}$, em uma tabela desenhada numa janela de figura do matlab, como apresentado na Tabela 3. Os ajustes da distribuição GEV referentes aos cinco valores de $\eta$ (LH0, LH1, LH2, LH3 e LH4) são plotados em um gráfico, juntamente com os dados observados de vazões (Figura 2). O melhor ajuste determinado a partir do menor valor do teste de Wang é exibido com os valores de cheias em outro gráfico (Figura 2); o mesmo procedimento pode ser aplicado para ajustar vazões mínimas. Na Figura 2 apresenta-se, ainda, um gráfico de vazões mínimas de sete dias, obtidos da mesma série de vazões diária observadas na estação fluviométrica sob o código 64685000 , localizada no Paraná.

As Tabelas 4 e 5 apresentam os resultados dos ajustes da distribuição GEV as 42 séries de cheia anual e de vazão mínima de 7 dias estudadas, nas quais são mostrados os valores dos três parâmetros da GEV, das taxas de momentos e dos testes de aderências aplicados, com os respectivos valores teóricos.
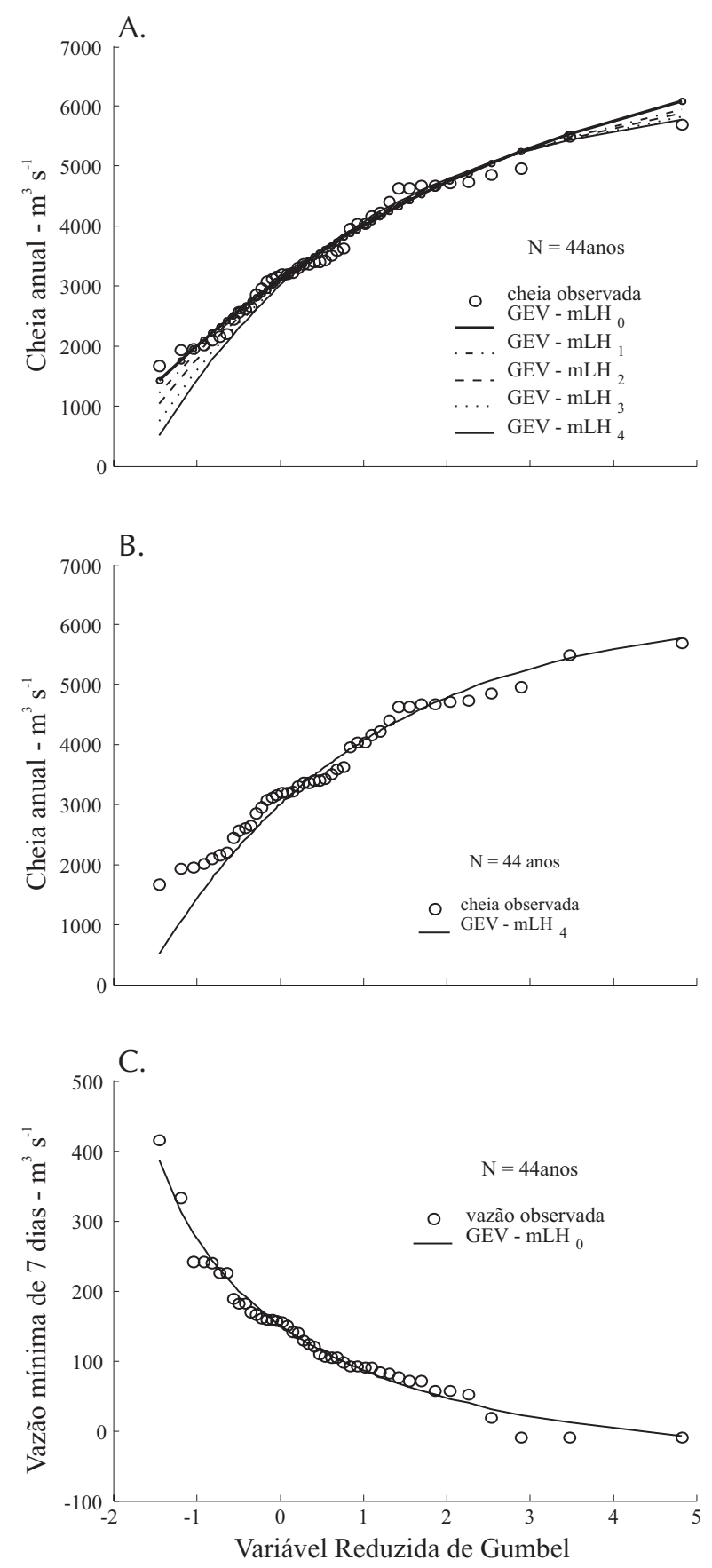

Figura 2. (A) Ajustes da distribuição GEV à série de cheia anual, aplicandose os cinco níveis de momentos LH; (B) Melhor ajuste da distribuição GEV à série de cheia anual e (C) Melhor ajuste da distribuição GEV à série de vazão mínima de sete dias

Tabela 3. Resultados do ajuste da distribuição GEV, fornecidos pela rotina em Matlab

\begin{tabular}{|c|c|c|c|c|c|c|c|c|}
\hline \multirow{2}{*}{ LH } & \multicolumn{3}{|c|}{ Parâmetros da distribuição GEV } & \multicolumn{3}{|c|}{ Taxas de momentos LH } & \multicolumn{2}{|c|}{ Testes estatísticos } \\
\hline & k & $\alpha$ & u & cv & ca & cc & $Z w$ & KS \\
\hline 0 & 0,2164 & 997,1 & 3105,8 & 0,1675 & 0,0382 & 0,0781 & 0,7295 & 0,0682 \\
\hline 1 & 0,2753 & 1065,4 & 3101,5 & 0,1117 & 0,0996 & 0,0602 & 0,6187 & 0,0682 \\
\hline 2 & 0,3142 & 1125,8 & 3081,8 & 0,0894 & 0,1227 & 0,0409 & 0,8683 & 0,0682 \\
\hline 3 & 0,3619 & 1216,5 & 3039,5 & 0,0766 & 0,1257 & 0,0416 & 0,6580 & 0,0909 \\
\hline 4 & 0,3954 & 1291,5 & 2998,0 & 0,0677 & 0,1277 & 0,0617 & 0,0820 & 0,0113 \\
\hline
\end{tabular}


Tabela 4. Ajustes da distribuição GEV às séries de cheia anual

\begin{tabular}{|c|c|c|c|c|c|c|c|c|c|c|c|c|c|}
\hline \multirow{2}{*}{$\begin{array}{l}\text { Código da } \\
\text { Estação }\end{array}$} & \multirow{2}{*}{ N } & \multicolumn{3}{|c|}{ Parâmetros da GEV } & \multicolumn{3}{|c|}{ Taxas de momentos } & \multirow{2}{*}{ LH } & \multirow{2}{*}{$\begin{array}{c}\text { W } \\
\text { ajuste }\end{array}$} & \multirow{2}{*}{$\begin{array}{c}\chi^{2} \\
\text { ajuste }\end{array}$} & \multirow{2}{*}{$\begin{array}{c}\chi^{2} \\
\text { teórico }\end{array}$} & \multirow{2}{*}{$\begin{array}{c}\text { Ks } \\
\text { ajuste }\end{array}$} & \multirow{2}{*}{$\begin{array}{c}\text { Ks } \\
\text { teórico }\end{array}$} \\
\hline & & k & $\alpha$ & u & cv & ca & cc & & & & & & \\
\hline 64231000 & 13 & $-0,41$ & 57,70 & 122,00 & 0,27 & 0,49 & 0,31 & 2 & 0,05 & 2,00 & 3,84 & 0,15 & 0,36 \\
\hline 64242000 & 20 & $-0,02$ & 133,00 & 108,00 & 0,19 & 0,30 & 0,12 & 4 & 0,45 & 1,64 & 3,84 & 0,25 & 0,29 \\
\hline 64360000 & 52 & $-0,12$ & 96,80 & 183,00 & 0,30 & 0,24 & 0,18 & 0 & 0,18 & 0,29 & 7,81 & 0,04 & 0,23 \\
\hline 64362000 & 21 & 0,21 & 231,90 & 468,00 & 0,15 & 0,13 & 0,12 & 1 & 0,28 & 1,71 & 3,84 & 0,10 & 0,28 \\
\hline 64370000 & 65 & 0,20 & 380,90 & 521,00 & 0,20 & 0,14 & 0,09 & 1 & 0,29 & 4,94 & 7,81 & 0,08 & 0,20 \\
\hline 64382000 & 19 & $-0,23$ & 192,20 & 342,00 & 0,34 & 0,33 & 0,18 & 0 & 0,65 & 1,67 & 3,84 & 0,11 & 0,30 \\
\hline 64390000 & 58 & 0,02 & 205,00 & 335,00 & 0,31 & 0,16 & 0,09 & 0 & 1,39 & 3,94 & 7,81 & 0,07 & 0,21 \\
\hline 64440000 & 25 & $-0,12$ & 39,50 & 95,00 & 0,19 & 0,31 & 0,18 & 1 & 0,05 & 0,15 & 5,99 & 0,12 & 0,26 \\
\hline 64442800 & 17 & 0,22 & 15,10 & 85,00 & 0,07 & 0,13 & 0,10 & 1 & 0,04 & 0,97 & 3,84 & 0,06 & 0,32 \\
\hline 64447000 & 22 & 0,52 & 490,00 & 226,00 & 0,12 & 0,08 & $-0,03$ & 4 & 0,94 & 0,29 & 3,84 & 0,22 & 0,27 \\
\hline 64450000 & 28 & $-0,30$ & 15,60 & 22,00 & 0,42 & 0,37 & 0,19 & 0 & 1,14 & 0,48 & 5,99 & 0,07 & 0,25 \\
\hline 64460000 & 27 & 0,01 & 101,80 & 26,00 & 0,22 & 0,29 & 0,08 & 4 & 1,15 & 0,40 & 5,99 & 0,17 & 0,25 \\
\hline 64460000 & 20 & $-0,01$ & 49,90 & 91,00 & 0,17 & 0,28 & 0,15 & 2 & 0,01 & 0,17 & 3,84 & 0,10 & 0,29 \\
\hline 64465000 & 66 & $-0,09$ & 229,60 & 498,00 & 0,27 & 0,23 & 0,14 & 0 & 0,89 & 1,47 & 7,81 & 0,05 & 0,20 \\
\hline 64475000 & 31 & $-0,32$ & 39,50 & 79,00 & 0,34 & 0,39 & 0,33 & 0 & 0,82 & 1,50 & 5,99 & 0,10 & 0,24 \\
\hline 64477600 & 17 & 0,53 & 189,90 & 141,00 & 0,10 & 0,07 & $-0,01$ & 4 & 0,49 & 0,34 & 3,84 & 0,18 & 0,32 \\
\hline 64501000 & 20 & 0,52 & 1417,60 & 1542,00 & 0,10 & 0,06 & $-0,03$ & 3 & 0,86 & 0,31 & 3,84 & 0,20 & 0,29 \\
\hline 64507000 & 65 & 0,16 & 1402,30 & 1220,00 & 0,15 & 0,23 & 0,07 & 4 & 1,28 & 0,43 & 7,81 & 0,23 & 0,20 \\
\hline 64508500 & 22 & 0,54 & 155,70 & 186,00 & 0,17 & $-0,03$ & 0,11 & 1 & 0,58 & 0,72 & 3,84 & 0,09 & 0,27 \\
\hline 64515000 & 23 & 0,28 & 3900,00 & 2655,00 & 0,14 & 0,18 & 0,11 & 4 & 0,26 & 0,44 & 3,84 & 0,20 & 0,27 \\
\hline 64550000 & 30 & $-0,07$ & 108,30 & 295,00 & 0,22 & 0,21 & 0,17 & 0 & 0,09 & 0,50 & 5,99 & 0,07 & 0,24 \\
\hline 64619950 & 13 & $-0,23$ & 96,80 & 215,00 & 0,29 & 0,32 & 0,24 & 0 & 0,08 & 0,44 & 3,84 & 0,08 & 0,36 \\
\hline 64620000 & 66 & $-0,34$ & 55,50 & 155,00 & 0,21 & 0,45 & 0,29 & 2 & 0,19 & 1,67 & 7,81 & 0,06 & 0,20 \\
\hline 64625000 & 30 & 0,08 & 332,40 & 782,00 & 0,23 & 0,12 & 0,11 & 0 & 0,32 & 2,62 & 5,99 & 0,07 & 0,24 \\
\hline 64655000 & 22 & 0,56 & 713,70 & 807,00 & 0,08 & 0,06 & 0,02 & 4 & 0,30 & 0,34 & 3,84 & 0,22 & 0,27 \\
\hline 64659000 & 22 & 0,56 & 713,70 & 807,00 & 0,08 & 0,06 & 0,02 & 4 & 0,30 & 0,34 & 3,84 & 0,22 & 0,27 \\
\hline 64673000 & 22 & $-0,31$ & 42,30 & 133,00 & 0,24 & 0,38 & 0,26 & 0 & 0,18 & 0,74 & 3,84 & 0,09 & 0,27 \\
\hline 64682000 & 20 & $-0,08$ & 15,00 & 40,00 & 0,23 & 0,22 & 0,10 & 0 & 0,89 & 1,67 & 3,84 & 0,10 & 0,29 \\
\hline 64685000 & 44 & 0,40 & 1291,50 & 2998,00 & 0,07 & 0,13 & 0,06 & 4 & 0,08 & 4,51 & 5,99 & 0,11 & 0,20 \\
\hline 64689000 & 13 & 0,44 & 578,20 & 3290,00 & 0,04 & 0,09 & 0,05 & 3 & 0,04 & 0,44 & 3,84 & 0,23 & 0,36 \\
\hline 64693000 & 22 & 0,46 & 1091,70 & 3784,00 & 0,05 & 0,08 & 0,06 & 3 & 0,08 & 0,79 & 3,84 & 0,14 & 0,27 \\
\hline 64771500 & 20 & 0,37 & 342,60 & 1316,00 & 0,06 & 0,12 & 0,08 & 3 & 0,19 & 0,20 & 3,84 & 0,10 & 0,29 \\
\hline 64773000 & 18 & 0,01 & 181,00 & 304,00 & 0,14 & 0,29 & 0,12 & 4 & 0,37 & 1,08 & 3,84 & 0,29 & 0,31 \\
\hline 64775000 & 29 & 0,38 & 405,20 & 697,00 & 0,08 & 0,14 & 0,06 & 4 & 0,19 & 3,97 & 5,99 & 0,21 & 0,24 \\
\hline 64785000 & 29 & 0,11 & 30,50 & 93,00 & 0,13 & 0,18 & 0,11 & 1 & 0,17 & 0,70 & 5,99 & 0,07 & 0,24 \\
\hline 64790000 & 31 & 0,01 & 38,70 & 75,00 & 0,13 & 0,29 & 0,16 & 4 & 0,12 & 0,41 & 5,99 & 0,10 & 0,24 \\
\hline 64795000 & 26 & 0,42 & 1246,50 & 2459,00 & 0,07 & 0,12 & 0,05 & 4 & 0,22 & 0,58 & 5,99 & 0,12 & 0,26 \\
\hline 64799500 & 31 & 0,01 & 38,70 & 75,00 & 0,13 & 0,29 & 0,16 & 4 & 0,12 & 0,41 & 5,99 & 0,10 & 0,24 \\
\hline 64810000 & 34 & $-0,12$ & 35,90 & 126,00 & 0,15 & 0,31 & 0,16 & 1 & 0,44 & 1,20 & 5,99 & 0,09 & 0,23 \\
\hline 64815000 & 34 & $-0,12$ & 35,90 & 126,00 & 0,15 & 0,31 & 0,16 & 1 & 0,44 & 0,24 & 5,99 & 0,09 & 0,23 \\
\hline 64830000 & 28 & 0,18 & 750,00 & 2664,00 & 0,09 & 0,18 & 0,07 & 2 & 0,51 & 3,84 & 5,99 & 0,11 & 0,25 \\
\hline 64843000 & 73 & $-0,16$ & 3045,90 & 17791,00 & 0,10 & 0,35 & 0,21 & 2 & 0,10 & 0,95 & 7,81 & 0,05 & 0,19 \\
\hline
\end{tabular}

$\mathrm{K}$ - parâmetro de forma; $\alpha$ - parâmetro de escala; u - parâmetro de posição; cv - coeficiente de variação; ca - coeficiente de assimetria; cc - coeficiente de curtose; Zw - teste de Wang, com Zw $(0,05)$ teórico $=1,96 ; \mathrm{Ks}$ - teste de Kolmogoroc-Smirnov, com Ks teórico de 0,05 de significância

Os ajustes da distribuição GEV aos dados de eventos hidrológicos extremos, referentes aos 5 níveis de combinações lineares $(\eta)$, dependendo do comportamento dos dados, podem resultar em valores de $\mathrm{k}>0$ ou $\mathrm{k}<0$ para os diferentes valores de $\mathrm{h}$, definindo o tipo de valor extremo (VEI, VEII ou VEIII); na prática, quando $-0,04<\mathrm{k}<0,04$, o ajuste se aproxima consideravelmente da distribuição Gumbel; Para $\eta=0$, o ajuste de momentos LH corresponde aos momentos L que atribui o mesmo peso para os dados durante o processo de ajuste; à medida que o valor de $\eta$ aumenta, os valores amostrais mais altos recebem maiores ponderações.

Na Figura 3 são apresentados os diagramas de momentos LH referentes ao ajuste da distribuição GEV às séries de cheias anuais e de vazões mínimas de sete dias observadas nas estações fluviométricas consideradas, como forma de mostrar a utilidade desta ferramenta para avaliar a qualidade dos ajustes obtidos através da distribuição GEV e momentos LH, a qual é possibilitada com o uso do algoritmo proposto.

Os testes de Wang e de Kolmogorov-Smirniv aplicados indicam que os ajustes da distribuição GEV às séries de cheia anual e de vazão mínima, foram aceitos com $5 \%$ de significância, ocorrendo as três formas de valores extremos. O teste proposto por Wang (1998) mostrou-se eficiente como teste de aderência aplicado às séries de cheia anual, em que os melhores ajustes ocorreram com os momentos LH, variando de $0 \mathrm{a} 4$; o mesmo não foi aplicado às séries de vazão 
Tabela 5. Ajustes da distribuição GEV às séries de vazão mínima de 7 dias

\begin{tabular}{|c|c|c|c|c|c|c|c|c|c|}
\hline \multirow[t]{2}{*}{ Estação } & \multirow[t]{2}{*}{$\mathbf{N}$} & \multicolumn{3}{|c|}{ Parâmetros da GEV } & \multicolumn{3}{|c|}{ Taxas de momentos LH } & \multicolumn{2}{|c|}{ Teste Kolmogorov } \\
\hline & & $k$ & $\alpha$ & $u$ & cv & ca & CC & Ajuste & Teórico \\
\hline 64231000 & 13 & 0,2640 & 3,4920 & 9,2470 & 0,1900 & 0,0116 & 0,1966 & 0,2300 & 0,3600 \\
\hline 64242000 & 20 & $-0,1290$ & 2,8960 & 10,130 & 0,1878 & 0,2535 & 0,2861 & 0,1500 & 0,2900 \\
\hline 64360000 & 52 & $-0,0420$ & 3,4210 & 7,1990 & 0,2649 & 0,1955 & 0,1255 & 0,0600 & 0,1900 \\
\hline 64362000 & 21 & 0,2520 & 5,8750 & 13,154 & 0,2206 & 0,0181 & 0,0225 & 0,1000 & 0,2800 \\
\hline 64370000 & 65 & $-0,0920$ & 5,4120 & 11,483 & 0,2716 & 0,2281 & 0,1535 & 0,0500 & 0,1700 \\
\hline 64382000 & 19 & 0,0980 & 3,3850 & 7,6220 & 0,1570 & 0,1903 & 0,1960 & 0,0500 & 0,3000 \\
\hline 64390000 & 58 & $-0,1490$ & 2,5500 & 5,6300 & 0,2745 & 0,2669 & 0,2236 & 0,0700 & 0,1800 \\
\hline 64440000 & 25 & 0,0450 & 1,4600 & 4,2050 & 0,1951 & 0,1401 & 0,0298 & 0,1200 & 0,2600 \\
\hline 64442800 & 17 & $-0,1170$ & 2,0040 & 4,1280 & 0,2825 & 0,2453 & 0,2875 & 0,1800 & 0,3200 \\
\hline 64447000 & 22 & 0,0990 & 11,063 & 22,115 & 0,2564 & 0,1069 & 0,1508 & 0,1400 & 0,2800 \\
\hline 64460000 & 27 & 0,0180 & 1,144 & 2,3260 & 0,2629 & 0,1568 & 0,1704 & 0,1100 & 0,2500 \\
\hline 64465000 & 66 & 0,0300 & 15,232 & 32,217 & 0,1744 & 0,2265 & 0,1843 & 0,0900 & 0,1700 \\
\hline 64475000 & 31 & $-0,1400$ & 1,5440 & 3,3150 & 0,2783 & 0,2608 & 0,1055 & 0,1000 & 0,2400 \\
\hline 64477600 & 17 & $-0,1180$ & 4,6190 & 5,6260 & 0,4064 & 0,2462 & 0,1314 & 0,0600 & 0,3200 \\
\hline 64501000 & 20 & 0,1140 & 38,337 & 100,823 & 0,2030 & 0,0978 & 0,1904 & 0,1000 & 0,2900 \\
\hline 64507000 & 65 & $-0,0520$ & 36,781 & 84,940 & 0,2479 & 0,2021 & 0,1352 & 0,0500 & 0,1700 \\
\hline 64508500 & 22 & 0,0830 & 1,963 & 4,366 & 0,2368 & 0,1165 & 0,1374 & 0,0900 & 0,2800 \\
\hline 64515000 & 23 & $-0,4380$ & 59,346 & 359,917 & 0,1630 & 0,5013 & 0,3134 & 0,0900 & 0,2700 \\
\hline 64550000 & 30 & $-0,0790$ & 10,089 & 32,145 & 0,1226 & 0,3246 & 0,1931 & 0,0700 & 0,2400 \\
\hline 64619950 & 13 & 0,0270 & 1,9370 & 4,0310 & 0,2570 & 0,1512 & $-0,0149$ & 0,0800 & 0,3600 \\
\hline 64620000 & 66 & 0,0500 & 1,5080 & 3,0550 & 0,2594 & 0,1369 & 0,0653 & 0,0600 & 0,1700 \\
\hline 64625000 & 30 & 0,0770 & 3,4380 & 8,3560 & 0,2209 & 0,1204 & 0,1486 & 0,1000 & 0,2400 \\
\hline 64659000 & 22 & $-0,0180$ & 2,7630 & 4,4660 & 0,3188 & 0,1801 & 0,0906 & 0,0900 & 0,2800 \\
\hline 64673000 & 22 & 0,0550 & 4,4580 & 12,873 & 0,1935 & 0,1336 & 0,2346 & 0,1800 & 0,2800 \\
\hline 64682000 & 20 & $-0,3190$ & 1,7620 & 8,1760 & 0,1477 & 0,4419 & 0,2995 & 0,0500 & 0,2900 \\
\hline 64685000 & 44 & $-0,0690$ & 58,526 & 114,501 & 0,2845 & 0,2128 & 0,1646 & 0,0700 & 0,2000 \\
\hline 64689000 & 13 & $-0,4170$ & 40,765 & 197,494 & 0,2009 & 0,4667 & 0,3248 & 0,1500 & 0,3600 \\
\hline 64693000 & 22 & $-0,3420$ & 44,368 & 193,374 & 0,1970 & 0,4082 & 0,3568 & 0,0900 & 0,2800 \\
\hline 64771500 & 20 & 0,0740 & 7,4800 & 11,787 & 0,3119 & 0,1220 & 0,0982 & 0,1000 & 0,2900 \\
\hline 64775000 & 29 & 0,2710 & 5,6550 & 9,6210 & 0,1271 & 0,1427 & 0,1248 & 0,1000 & 0,2400 \\
\hline 64785000 & 29 & 0,2180 & 5,4410 & 12,073 & 0,2247 & 0,0375 & 0,0637 & 0,0700 & 0,2400 \\
\hline 64790000 & 31 & 0,0260 & 2,2250 & 5,2690 & 0,2319 & 0,1521 & 0,1318 & 0,1000 & 0,2400 \\
\hline 64795000 & 26 & 0,0470 & 26,769 & 48,956 & 0,2815 & 0,1387 & 0,1580 & 0,0800 & 0,2600 \\
\hline 64810000 & 34 & 0,1350 & 6,0130 & 22,295 & 0,1490 & 0,0855 & 0,1534 & 0,0900 & 0,2300 \\
\hline 64830000 & 28 & 0,0560 & 53,678 & 132,379 & 0,2207 & 0,1334 & 0,1339 & 0,0700 & 0,2500 \\
\hline 64843000 & 77 & $-0,1130$ & 1503,02 & 4107,24 & 0,2260 & 0,2420 & 0,0860 & 0,0600 & 0,1500 \\
\hline 64841000 & 73 & $-0,0870$ & 1409,00 & 4032,00 & 0,1992 & 0,2260 & 0,0783 & 0,0300 & 0,1600 \\
\hline
\end{tabular}

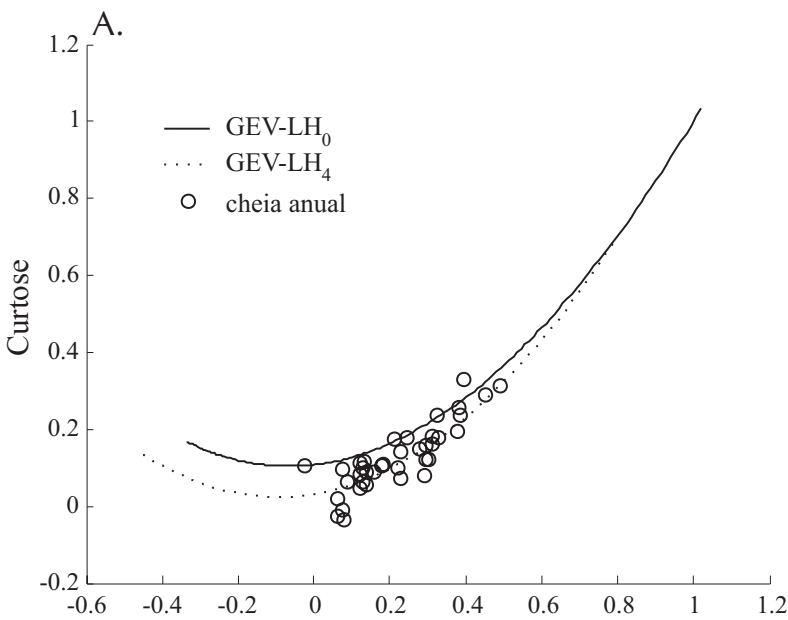

B.

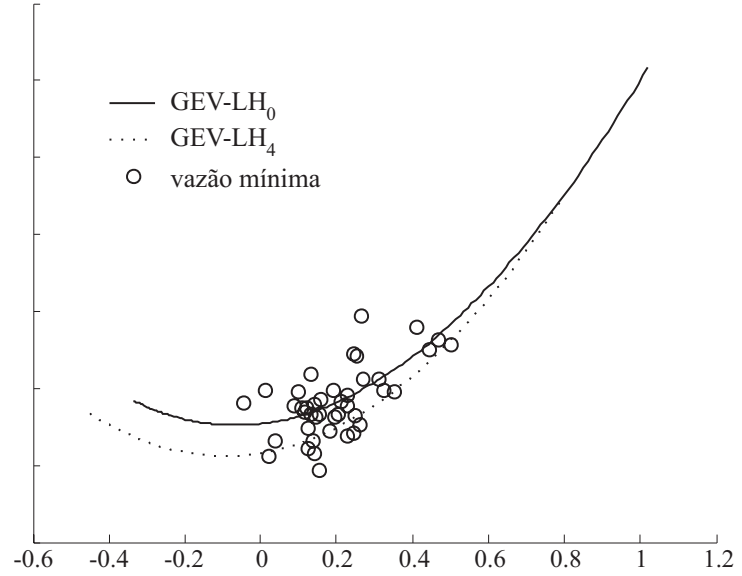

Assimetria

Figura 3. A. Diagrama de momentos LH para as cheias anuais e B. Diagrama de momentos LH para as vazões mínimas de 7 dias 
mínimas visto que os coeficientes apresentados na Tabela 2 se referem a valores extremos máximos.

\section{CONCLUSÕES}

1. A distribuição GEV e os momentos LH forneceram ajustes adequados das cheias anuais e das vazões mínimas, conforme os testes de aderência de Wang e de KolmogorovSmirnov, com 5\% de significância, aplicados através da rotina proposta em matlab.

2. O programa fornece, como resultados, os três parâmetros da distribuição GEV, às razões de momentos LH que permitem traçar o diagrama de momentos LH, além dos valores do teste de Wang (1998) que avalia a qualidade do ajuste para cada nível LH.

3. O teste de qualidade de ajuste proposto por Wang (1998) indicou os melhores ajustes, seguindo os resultados fornecidos pelo teste de Kolmogorov-Smirnov, referentes às séries de cheia anual modeladas.

\section{LITERATURA CITADA}

Chowdhury, J. U.; Stedinger, J. R.; Lu, L. Goodness-of-fit test for regional generalized extreme value flood distributions. Water Resources Research, Washington, v.27, n.7, p.17651776, 1991.

Fisher, R. A.; Tippett, L. H. C. Limiting forms of the frequency distribution of the largest or smallest member of a sample. Proceedings of the Cambridge Philosophical Society, Cambridge, v.24, n.2, p.180-190, 1928.

Gumbel, E. J. Statistics of extreme. New York: Columbia University Press, 1958, 396p.
Hosking, J. R. M.; Algorithm AS215: maximum-likelihood estimation of the parameter of the generalized extrem-value distribution. Journal of the Royal Statistical Society: Series C (Applied statistics), London, v.34, n.3, p.301-310, 1985.

Hosking, J. R. M. L-moments: analysis and estimation of distribution using linear combinations of order statistics, Journal of the Royal Statistical Society: Series B (Statistical methodology), London, v.52, n.2 p.105-124, 1990.

Hosking, J. R. M.; Wallis, J. R.; Wood, E. F. Estimation of the generalized extreme value distribution by the method of probability weighted moments. Technometrics, Alexandria, v.27, n.3, p.251-261, 1985.

Jenkinson, A. F. The frequency distribution of the annual maximum (or minimum) of meteorological elements, Quarterly Journal of the Royal Meteorological Society, London, v.81, n.551.501.45, p.158-171, 1955.

Martins, E. S.; Stedinger, J. R. Generalized maximum-likelihood generalized extreme-value quantile estimators for hydrologic data. Water Resources, Research, Washington, v.36, n.3, p.737-744, 2000.

Otten, A.; van Monfort, M. A. J. Maximum-likelihood estimation of the general extreme-value distribution parameters. Journal of Hydrology, Amsterdam, v.47, p.187-192, 1980.

Prescott, P.; Walden, A. T. Maximum - likelihood estimation of the parameters of the generalized extreme - value distribution. Biometrika, Oxford, v.67, p.723-724, 1980.

Queiroz, M. M. F. de Análise de cheias anuais segundo distribuição generalizada: EESC/USP, 2002. 254p. Tese Doutorado

Wang, Q. J. LH moments for statistical analysis of extreme events, Water Resources Research, Washington, v.33, n.12, p.28412848, 1997.

Wang, Q. J. Approximate goodness-of-fit test of fitted generalized extreme value distribution using LH moments, Water Resources Research, Washington, v.34, n.12, p.3497-3502, 1998. 